\title{
Microtubule dynamics in cytoskeleton, neurodegenerative and psychiatric disease
}

\author{
Simone MORTAL * \\ Neuroscience Area, International School for Advanced Studies (SISSA), 34136 Trieste, Italy \\ *Correspondence: smortal@sissa.it \\ https://doi.org/10.37175/stemedicine.v2i6.81
}

\begin{abstract}
Microtubules (MTs) are fundamental polymers composed by $\alpha$ and $\beta$ tubulin, they provide integrity to neuronal cell and are necessaries in intracellular trafficking and organization. The extension and retraction of MTs occur with the addition or removal of $\alpha$ and $\beta$ tubulin subunits and the binding with microtubule associated proteins (MAPs) that selectively target specific tubulin regions, manipulating the MT dynamics and function. Altered MT homeostasis can compromise the function of MTs in the structural integrity and axonal transport inside the neuron. Here I review the evidence of MT anomalies in several neurodegenerative diseases, including Alzheimer's disease, Parkinson disease, amyotrophic lateral sclerosis and traumatic brain injury and psychiatric disorders, such as depression, schizophrenia, and bipolar disorder. The focus of this review is to point out which can be the impact of MT issues in the major neurodegenerative diseases and discuss which MT abnormalities can lead to psychiatric illnesses.
\end{abstract}

Keywords: Microtubules · MAPs · Alzheimer's disease · Amyotrophic lateral sclerosis · Schizophrenia Psychiatric disorders · Neurodegenerative diseases

\section{Introduction}

During the neuronal development stages, aside from the actin cytoskeleton, the assembly, organization and remodelling of the microtubule (MT) cytoskeleton is essential (1-3). MTs compose the rails for intracellular transport, they can act as signalling devices, generate cellular forces and mediate vesicular release (4-7).

The MT cytoskeleton is fundamental in neuronal development, this is highlighted by the wide range of nervous system abnormalities and several human neurodevelopmental disorders linked to altered MTmediated processes. In addition, there are several developmental problems that can be linked to mutations in MT-related genes that encode for microtubule-associated proteins (MAPs), MT motor associated regulators or MT severing proteins $(8,9)$.

Received: Feb 8, 2021; Accepted: Mar 22, 2021.

(c) The Author(s). 2020 This is an Open Access article distributed under the terms of the Creative Commons License (http://creativecommons.org/licenses/by/4.0/) which permits unrestricted use, distribution, and reproduction in any medium or format, provided the original work is properly cited.
In this review I examine how MTs are built, and which are the major proteins that interact with them. Then I analyse some of the most important neurodegenerative disorders exploring the interaction that connects disease with MT cytoskeleton. In the last part I discuss how anomalies in the MTs, or related proteins, can lead to psychiatric disease.

\section{Microtubule organization}

The MT structure is built from heterodimers of $\alpha$ and $\beta$ tubulin, that are bound in a head to tail relation to form polarized structures; these associate laterally to form a hollow tube, with a diameter of $25 \mathrm{~nm}$ (5). MTs are very dynamic structures, that continuously switch between elongation and disassembly, in a process called dynamic instability; this procedure allows individual MT to explore cellular regions and to retract if it does not find the proper environment (10)

MT function and dynamics are regulated by the properties of tubulin; the free tubulin binds GTP, which is hydrolysed after being incorporated into the MT structure. The growth of the MT is promoted by the GTP cap, in 
fact, GDP-tubulin tends to destabilize the MT architecture, consequently stable growth is believed to depend on the presence of a GTP-tubulin cap at the MT plus end (11). In this model, the loss of the cap will result in a collapse of the structure, called catastrophe (12).

At the end of the MT there is a minus end and a plus end, these ends can grow and depolymerize, but these two dynamics are completely different. Minus ends are stabilizes by minus end tracking proteins (-TIPs), they grow more slowly and undergoes catastrophe less rarely, not a lot is known about them, in fact they start to emerge recently (13). The plus end, terminate with $\beta$-tubulin, grows with polymerization of the subunits, undergoes catastrophe more frequently and is a crucial site for regulating MT dynamics (14).

Plus end tracking proteins (+TIPs) concentrate at the ends of growing MTs and control different aspects of its progress, like recruiting tubulin dimers and increasing the rate of tubulin addition to growing tips (15). Nevertheless, the polymerization of microtubule based structures not only depends on +TIPs, but also requires many other factors, like MAPs, motor proteins and tubulin isotypes (5).

Concentrating on the different ways of action, five groups of proteins can be assumed from the microtubule related proteins. Firstly, proteins that bind to the MT ends and can regulate their dynamics, this group contains the +TIPs and minus end targeting proteins (-TIPs) $(5,14)$. Then, the group of proteins that bindS to the MT lattice and can stabilize or crosslink MTs $(16,17)$. The third group includes proteins that modulate the MT abundance, enzymes that sever MTs and regulators of the nucleation $(18,19)$. The fourth group contains the kinesin and dynein families that generate forces and move directionally along MTs (20). The last group comprises tubulin folding cofactors and tubulin modifying enzymes that can generate distinct MT subtypes through post translational modifications (21) (Figure 1).

\section{The Neuronal MT Cytoskeleton}

The MTs of neurons are involved in the morphological changes during the different phases during their development, in the intracellular transport and during synapse growth.

Neurons need to have an active and efficient transport mechanism, to properly distribute many cellular components and establish signalling pathways. The motor protein families that play this role are Kynesins and dyneins, they travel along the neuronal MTs carrying many types of neuronal cargo: neurotransmitter receptors, synaptic vesicles, cell adhesion molecules, organelles, cell signalling molecules and mRNAs. In addition, cargo adaptor proteins, regulatory molecules and MT cytoskeleton have an important role in the delivering of different cargos in the correct location (20).

MTs are fundamental in the morphological transitions that occur during neuronal development: the neurite initiation, migration, polarization and differentiation.

Neuronal migration is a complex sequence of motile and morphogenetic events, in which neurons extend primary processes and translocate the nucleus into this processe. These movements are driven by actin and MTs filaments, the first promotes neuronal migration by propulsive contractions at the cell rear and MTs which are anchored to the centrosome extend the leading process and form a cage-like structure around the nucleus. Cytoskeletal forces in the leading edge can pull the centrosome into the proximal part of the leading process and move the nucleus in the direction of cell migration (22).

Neurite initiation and outgrowth begins with the breakage of the circular shape of new-born neurons by emerging neurites. These newborn neurites are composed by bundled MTs and a growth cone which mediates the pushing and pulling forces that provide to membrane protrusion $(23,24)$.

MT stabilization plays a fundamental role in axon differentiation during the neuronal polarization. The increased MTs stability leads to kinesin mediated flow and contributes to determining the future axon formation. The complete mechanism of axon differentiation remains unknown, but internal signals, like centrosome localization, Golgi position and cytoskeleton architecture, could introduce a local imbalance inside the MT network that leads to stabilize MTs in only one of the many neurites $(25,26)$.

During axonal elongation the MT cytoskeleton participates in functional interactions with actin and adhesion complex, that with +TIPs can modulate the MT properties and stability (25). In addition to MT polymerization, recent studies found that the translocation of whole MT bundles into the axon contribute to axon elongation, that is presumably generated by molecular motors $(27,28)$.

Dendritic spine morpho dynamic and synapse functioning are linked directly to MT dynamics, in fact, evidences suggest that MTs are associated with transient changes in spinal shape, such as the formation and enlargement of the spine. MTs that entered into spines are regulated by the neuronal activity and brain derived neurotrophic factor (BDNF), these MTs with MT dependent motors are responsible to drive postsynaptic cargoes into spines (29).

MTs cytoskeleton is organized in bundles and axonal cross sections are usually composed by 10-100 MTs. In several cell types, MTs are nucleated at the microtubule organizing centre (MTOC), such as the centrosome, but they can be generated even in other different places, such as the Golgi apparatus or along existing MTs, where not all the minus ends are directed towards the MTOC (30,31). In new-born neurons the centrosome first acts as an active MTOC, but with time this activity is completely lost. Super resolution studies and electron microscopy have shown that MTs are not anchored to the centrosome and often in mature neurons there are free ends (Figure 2).

Because most MTs do not emerge from MTOC, their relative orientations can be different. Electron microscopy with hook decoration technique showed that MTs orientations in axons and dendrites have two different patterns (32). In axons is possible to observe uniformly plus end out oriented MTs, while in proximal dendrites 


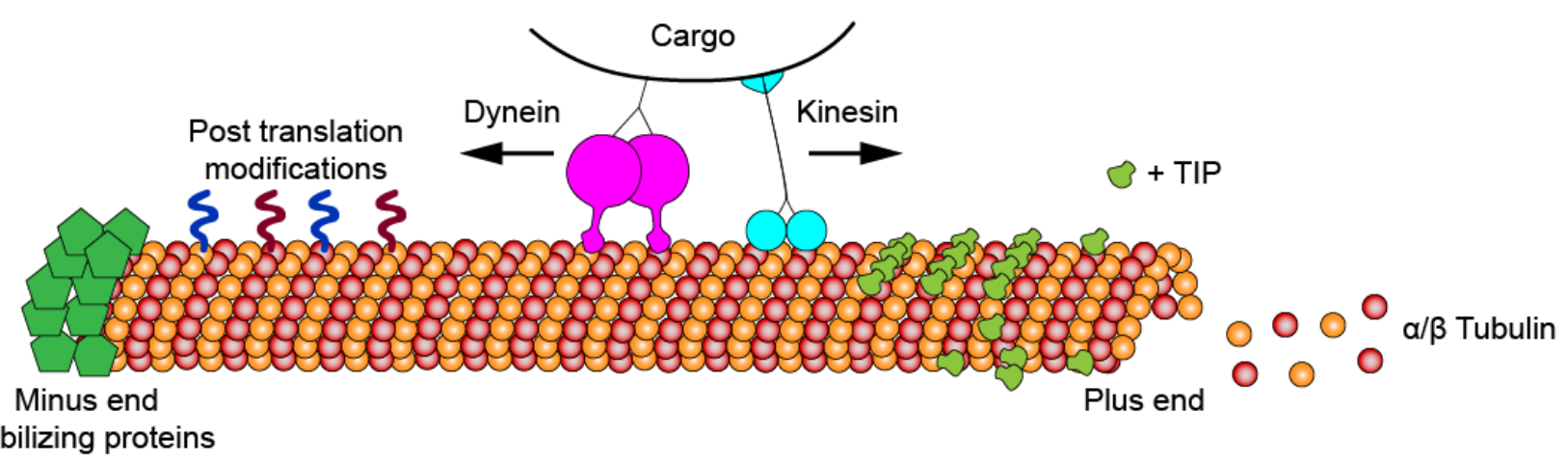

Figure 1. The cartoon illustrates different MAPs that interact with the MT.

the MTs orientations are non-uniformly, in fact MTs are half plus end out and half minus end out oriented.

This different distribution in MTs orientation contributes to the differential trafficking in axons or dendrites (33).

The selective presence of minus end out oriented in dendrites allow dyneins to selectively transport cargoes in dendrites. On the other hand, kinesin 1 has been shown to selectively transport cargoes into the axon, where MTs are composed by almost all plus end out oriented $(25,34)$. This separation of duties is possible because kinesin move along the MTs towards the plus end while dynein move to the minus end (35).

\section{Microtubule Associated Proteins}

The microtubule dynamics cannot be efficient and determined only by polymerized tubulin, but need specific proteins that interacts with tubulin - the microtubule associated proteins (MAPs). Depending on their role, MAPs can be categorized into motile MAPs, motor proteins that generate movements and forces $(36,37)$; enzymes that cut or depolymerize tubulin (38); nucleators (39); end-binding proteins that bind the minus or plus ends of microtubules (13); and the structural MAPs. While, the first four categories of MAPs are well defined by their functions, the last group bind microtubules to stabilize them, but there is not a clear and broad view on their functions (40).

Structural MAPs are a large category, that includes different proteins all able to decorate the MT cytoskeleton, but only some of them, such as tau, induce microtubule bundling $(41,42)$. A possible mechanism on how MAPs can directly participate in microtubule bundling was recently proposed: MAPs are proteins rich in positive charged ammino acids, the abundance of this charges neutralize the negative charges of the C-terminals of tubulins (43). This process reduces the electrostatic repulsion between MTs and allow the bundling them.

In particular, MAP2 is extremely present in brain and its function is directly related to neuronal plasticity. It has multiple isoforms, the high molecular weight (HMWMAP2), MAP2A and MAP2B selectively expressed in neurons and the low molecular weight (LMWMAP2), MAP2C and MAP2D expressed in neurons and glia $(44,45)$. MAP2 induce characteristic changes in microtubule structure, increase the MTOC-independent microtubule polymerization and is a cross-linker of tubulin filaments $(46,47)$. Reduced levels of MAP2, can be compensate by MAP1b, but the deletion of both genes leads to perinatal lethality (47).

Several different MAPs can potentially coexist on one MTs and a big number of binding sites are present on the microtubule surface. MAPs bind the microtubules for different functions, like binding with specific proteins or cross linking of different MTs. Although a common effect of several MAPs is to reduced microtubule tendency to depolymerize, however is important to remember that MAPs are not merely sticked to MTs, but they dynamically bind and unbind MTs.

\section{Microtubules in Neurodegeneration}

Several neurodegenerative disorders are characterized by altered axonal transport, that can cause neuronal damages and death. Though different causes can contribute to damage the axonal transport, in many cases appear that transport deficits result from reduced MT stability (48). Here, I review evidences of MT instability in some of the most common neurodegenerative disease, such as Alzheimer's Disease, Parkinson's Disease, traumatic brain injury and amyotrophic lateral sclerosis.

\section{Microtubules dysfunction in Alzheimer's Disease and Tauopathies}

Tauopathies are neurodegenerative disorders characterized by the deposition of insoluble fibrils of hyperphosphorylated tau, that are called neurofibrillary tangles (NFTs) and are localized in the neuronal soma or dendrites. Alzheimer disease (AD) is the most notorious disorder among tauopathies, but it is not only due by tau, in fact this disorder is a mixed proteinopathy, where amyloid beta $(\mathrm{A} \beta)$ peptide, $\alpha$-synuclein and TDP-43 are usually involved.

Tau protein is thought to play a fundamental role in MT properties and functions: the hyperphosphorylation of tau reduces the binding affinity to MT and lead to form insoluble fibrils. It is believed that Tau - mediated toxicity is due to the formation misfolded insoluble fibrils and/ or altered properties of MT that result in a release of tau form MT and a subsequent aggregation into inclusions. 


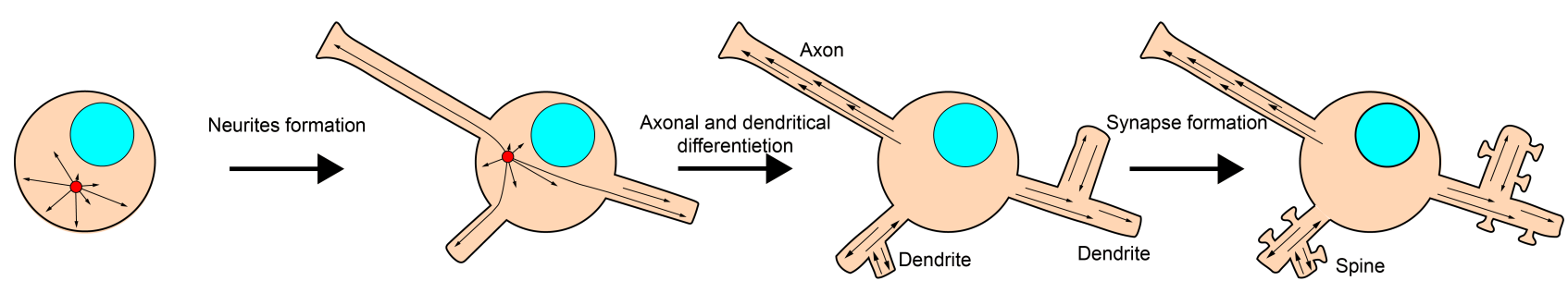

Figure 2. MT changes during neuronal development. After their final division, neurons transit through many developmental stages and the MT cytoskeleton has a crucial role at all the different stages. The MT cytoskeletal organization changes from a radially centrosome-based with largely plus-end out-oriented network to an acentrosomal network with uniform orientations in the axon and mixed orientation in dendrites.

The contribute of tau loss of function in neurodegeneration of tauopathies, is confirmed by observations of reduced number of MT in AD brains and the decreased concentration of acetylated $\alpha$-tubulin, that is an hallmark of stable MTs. Furthermore, studies on mouse models for NTFs tauopathies revealed abnormalities in MT, such as the reduction in MT concentration, the increased MT dynamicity and reduced axonal transport. These results have led to consider agents that increased MT stability, such as drugs for the treatment of cancer (49), as potential candidates for treatment of AD and tauopathies (50).

\section{Traumatic brain injury}

There are many observations that repetitive traumatic brain injuries (TBI) (51) induce chronic traumatic encephalopathy (CTE), characterized by behavioural anomalies and neuronal loss (52). In particular, brains present neurons with diffuse axonal damage, large breakage of the MT cytoskeleton and the loss of axonal transport (53-55). Interestingly, repeated TBI can lead to develop tau pathology that starts to spread out from cortical sulci; this can be explained by the relationship between tauopathies and reduction of MT stability and axonal transport $(53,56)$. Moreover, TBI can outcome in $\mathrm{AD}$ or other neurodegenerative symptoms (57). This suggests that MT stabilizing drugs can be effective on the neurodegenerative conditions caused by TBI/CTE. On this way the MT dysfunctions are becoming one of the distinctive features of TBI and on the therapy front, good results are coming from MT stabilizers drugs such as epoD and paclitaxel (58-60)

\section{Parkinson's disease}

Parkinson's disease (PD) is a brain disorder which provide locomotor impairments, that is due to the loss of dopaminergic neurons in the substantia nigra pars compacta. Pathological neurons of this region present intra neuronal inclusions of $\alpha$-synuclein called Lewi bodies. As in $\mathrm{AD}$, there are evidences of MT anomalies in PD brains that probably contribute to neurodegeneration. In fact, the extensive axonal arborization of the dopaminergic neurons of substantia nigra because makes this type of neurons particularly fragile in case of damaged of axonal transport. Studies on the relationship between $\alpha$-synuclein and MTs revealed that neurons overexpressing $\alpha$-synuclein present MT dysfunction, compromised MT dependent trafficking, Golgi fragmentation and neurite degeneration. Interestingly a possible involvement of hyperphosphorylated tau in MT instability was observed in PD models; with the involvement of kinase LRRK2 that is mutated in familiar cases of PD. This protein can phosphorylate tau bound to MTs, reducing the binding of tau with MTs and increasing the free tau inside the cell which can lead to pathology. Furthermore, the increased tau phosphorylation by mutated LRRK2 is probably due to an enhanced binding affinity between mutated LRRK2 and MTs. Moreover, overexpression of LRRK2 in transgenic mouse models with mutation A53T on $\alpha$-synuclein worsen the pathology and with an acceleration in $\alpha$-synuclein aggregation, that can be due to the damaged dynamics of MT. Therefore, alterations in MTs stability can cause an increase of both $\alpha$-synuclein and tau pathology.

Lastly decreased MT stability can also come from mutations on Parkin protein that cause familial PD. Studies on neurons derived from iPSC fibroblasts of patients with mutated Parkin protein showed a reduction in MTs stability and consequently a reduced neurite length and arborization. Notably, the altered morphology of this neurons can be repaired with the administration of pacilitaxel, a MT stabilizing drug (61).

However, action of Parkin protein on MT is not fully understood and interesting it can interact with HDAC6 (62), that can deacetylate MTs, but additional studies are needed to investigate the Parking effect on MT.

\section{Amyotrophic lateral sclerosis}

Amyotrophic lateral sclerosis (ALS) is a progressive nervous system disease of motor neurons (63). Why these neurons seem to be the more vulnerable to ALS is not completely clear, but MT instability and deficient axonal transport would be particularly destructive in motor neurons that have a morphology with longest axons in the body. In this way there are evidences that support the involvement of defective axonal transport in ALS (64). For example, dynactin is a protein that works in association with dynein, is very important in retrograde axonal transport, but when mutated in subunit p150glued can cause motor neuron disease and maybe ALS (65). Additionally, $10 \%$ of hereditary ALS cases present mutations superoxidase dismutase 1 (SOD1) gene, that participates anterograde mitochondrial transport inaxons (66). Experiments on transgenic mouse models with 
SOD1 mutated develop axonal transport deficits before the onset of the neurodegeneration $(67,68)$. In addition, these transgenic mice line present increased MT dynamicity that can be restored on normal levels with noscapine, can restore the axonal transport and decrease the MT turnover, with a reduced cell death (69). Moreover, other studies in ALS mouse models confirmed that mutated SOD1 increase the MT dynamicity, expanding the evidences that mutations in SOD1 perturb the MT homeostasis $(70,71)$.

As mutated SOD1 concerns a little fraction of ALS patients, evidence of altered MT dynamics and axonal transport are needed in non SOD1 ASL models to support the involvement of MTs in ALS. In this way, studies on mouse model for ALS (TDP-43) revealed that mutations on TDP-43, a DNA binding protein, are associated with axonal transport problems (72). TDP-43 is implicated in ALS, in fact it is the main protein found in ubiquitin positive inclusions that are characteristic of ALS pathology. In addition, mouse models TDP-43 have a reduction in mitochondrial motility, that can appear just before the onset of the first symptoms of ALS (73).

Nevertheless, the molecular mechanisms implicated are not fully understood and further experiments will be necessary to understand the molecular cascade that links TDP-43 and SOD1 with MT stability and ALS.

\section{Therapeutic Drugs}

As discussed herein, there are several evidences that the axonal transport deficit is involved in numerous neurodegenerative disease and this is probably induced by the altered MT stability. In these way MT stabilizing drugs were identified as potential candidates for treatment of neurodegenerative disease. MT stabilizing drugs, as it is known, can inhibit the cell division and thus are the ideal drugs to work in CNS and interact with neurons. Recent results with epothiloneD (epoD) a MT stabilizer show encouraging benefits in mouse models (74). In particular, different studies showed the efficacy of epoD in reducing the MT hyperdinamicity and neuronal loss. This drug is particularly effective in thaupaties and AD (75-77), but significant benefits were noted even for TBI and PD $(58,78)$. However, other drugs were studied in mouse models, such as dictyostatin, this drug have relevant benefits in the CNS, but complications in the gastrointestinal tract and mortality was observed on mouse models (79-82). This show that not all MT stabilizing drugs are equal, and that carefulness and further studies will be necessary to guarantee benefits without major side effects.

\section{Microtubules in Psychiatric Disease}

Cytoskeleton dysfunctions have been observed even in several neuropsychiatric disorders such as schizophrenia, major depressive disorder (MDD) and bipolar disorder. The hypothesis that altered MT dynamics leads to a disrupted synaptic connectivity is supported by clinical studies and animal models. Here, I present some findings that connects altered MTs homeostasis with psychiatric diseases.

\section{Tubulin Isotypes}

During human brain development various tubulin isotypes are expressed, the different isotypes can influence the structural and interaction properties of the MT and have different affinity with PMT modifications. In particular $\alpha_{1 \mathrm{~A}}, \beta_{\mathrm{II}}$ and $\beta_{\mathrm{III}}$ are the most present tubulin isotypes in the brain, while $\alpha_{1 \mathrm{~B}}, \alpha_{1 \mathrm{C}}, \beta_{\mathrm{I}}$ and $\beta_{\mathrm{IVb}}$ that are expressed in the whole body. Altered expression of tubulin isotypes were identified from proteomic studies in rodent models of depression. It has been noted that isolation rodent models have a reduction in $\alpha$-tubulin expression (83) and rats genetically sensitive to depression have a huge decrease in $\beta_{\text {IIA }}$ and $\beta_{\mathrm{V}}$ tubulin expression in hippocampus (84). These studies show that reduced $\alpha$ and $\beta$ tubulin expression in hippocampus is a hallmark of depression in rodents models.

It is possible to observe decreased tubulin isotypes even in humans, for example in patients with schizophrenia or bipolar disorder have a decreased expression of $\beta$-tubulin isotype in the prefrontal cortex (85). Coherent with this, gene expression analysis from patients with major depressive disorder, shows that TUBB4 and $T U B B 2 C$ isotypes are down-regulated in the dorsolateral prefrontal cortex (86). Taken together these data highlight that the expression of tubulin isotypes is significantly altered in clinical and animal models of psychiatric disorders, showing that distorted tubulin homeostasis is a characteristic these circumstances.

\section{Tubulin PTMs}

Post translational modifications of tubulin (PTMs) is a powerful mechanism to generate specific microtubule identities (87). These modifications which include acetylation/deacetylation, phosphorylation, tyrosination/ detyrosination, glutamylation and polyglycylation create a unique environment for the interaction of specific MAPs to the MTs (88).

Acetylation/deacetylation. Acetylation can be performed on both $\alpha$ and $\beta$ tubulin residues, where acetyl transferase $\alpha$ TAT and Atat- 2 act on the former and Sun acetyltransferase work on $\beta$-tubulin (88). On the other hand, deacetylation is performed by histone deacetylase 6 (HDAC6) and nicotine adenine dinucleotide-dependent deacetylase sirtuin-2 (SIRT2) (89).

The role of HDAC inhibitors as antidepressants is well known and it was largely tested on rodent models of depression (90). On this way, behavioural studies conducted on HDAC6 Knockout (KO) mice revealed that these mice express low anxiety, hyperactivity and low depressive phenotype (91). Furthermore, reduced expression of SIRT2 deacetylase is characteristic in rodent models of depression, suggesting that this deacetylase protein can be directly implicated in depressive behaviour (92).

Reduced levels of tubulin acetylation have been observed even in rats after social isolation (83), while they are increased in the hippocampus of rats after exposure to unpredictable chronic mild stress (93). Tubulin acetylation is also diminished after knockdown of Ulk4 (Unc-51 like kinase-4), a gene that has been funded deleted in 
schizophrenia and bipolar disorders(94). Mouse models with Ulk4 knockdown have reduced neurite length, diminished branching, and agenesis of the corpus callosum. Interestingly also, c-Jun NH2-terminal protein kinase (JNK) activity is reduced in these Ulk4 knockdown mice $(95)$. JNK is a protein that regulate the MT homeostasis and neurite complexity and it can represent a possible link between Ulk4 and MT integrity.

All these findings suggest that tubulin acetylation levels are altered in models of schizophrenia and depression. This can be the cause of abnormalities in the development of axons and dendrites and synaptic plasticity. Furthermore, kinases implicated in schizophrenia have a role in the pathway that controls MT stabilization, suggesting a link between pathological symptoms and the loss of cytoskeletal homeostasis.

Phosphorylation. The addition of a phosphate group results in a gain of negative charges that change the chemical properties of tubulin. Phosphorylation is performed on neuronal $\beta$ III tubulin and on $\alpha$-tubulin, this mechanism is particularly active during neuronal differentiation and helps the microtubule polymerization (96-98). Phosphorylation of tubulin induces conformational changes of MT, this can change the binding site for stabilizing drugs used in psychiatric disease such as valproate lithium and paliperidone, implying that this modifications are relevant in pathologies (99).

Tyrosination/detyrosination. Tyrosination is performed by tubulin tyrosine ligase (TTL) on $\alpha$-tubulin; tyrosinated tubulin increase microtubule dynamics while detyrosination enhance microtubule stability, these dynamics are important in axonal extension and transport (88). Detyrosination concerns the removal of Tyr from the C-terminal of $\alpha$-tubulin to expose a Glu residue. In this way, Glutamylation is done by tubulin tyrosine ligaselike (TTLL), that add chains of glutamates to the carboxy terminal of tubulin. This happens at high levels usually in brain. Polyglutamylation influence the surface charges on MTs modifying the binding properties to MAPs and motor proteins (100). It is known that damaged protein transport and the compromised binding of MAPs can contribute to pathology in schizophrenia (101). In fact, problems on the TTLL11 ployglutamylase gene are linked to schizophrenia and bipolar disorder $(102,103)$.

Polyglycylation. This modification is peculiar of cilia and flagella. It can be present on $\alpha$ and $\beta$ tubulin and the responsible enzymes are the members of the TTL family (104). Glycylation is important in stabilization and motility of ependymal cilia which plate ventricles of the brain (105), but it is interesting to note that in cilia effects of diverse neuropsychiatric risk genes converge (106).

\section{MAPs}

MAPs are fine regulators of the microtubule properties and function in neuronal network; therefore an imperfect function of these proteins can give rise to neurodegenerative, developmental and psychiatric disorders (29).

\section{MAPs in Psychiatric Disorders}

Dendritic arborization and spine density is directly related with MAPs (29), in fact reduced levels of these can lead to neurological disease $(107,108)$, intellectual disability (109), depression $(110,111)$ and schizophrenia (108). In case of depression the synapse loss damages the feedback loops and the adaptive response for stress (112). Spine loss in schizophrenia is associated with reduced MAP2 (113), suggesting a participation in the spine stabilization, suggesting a role for MT in in spine head stability. Crucial for synaptic disorders is also how MAP2 impact on CREB activity by binding PKA in dendrites (114). CREB controls the expression of BDNF, that is fundamental in synapse and neuronal survival.

\section{Loss of MAP2 immunoreactivity is an hallmark of schizophrenia}

In the last twenty years several studies were conducted post-mortem on schizophrenia patients and a common feature of the analysed brains were the reduced immunoreactivity of MAP2 (113). This was localized specifically in hippocampus and prefrontal cortex where it coincides with diminished dendrite ramification. The reduced immunoreactivity noted does not mean reduced MAP2, in fact proteomic analysis on schizophrenic or depressed patients show no altered levels of MAP2 protein (85). Consistent with this MAP2 mRNA is not altered in patients with schizophrenia (115), but the decreased immunoreactivity seems due to PTM of MAP2 (e.g., phosphorylation), that limit the epitope recognition. This modification probably can lead to an altered function of MAP2 and contribute to the typical disorders of psychiatric disease.

\section{MAP6}

Like MAP2, MAP6 stabilizes microtubules, binding together the nearby microtubules $(116,117)$. Mice MAP6 KO present severe symptoms associated with schizophrenia, that can be anxiety, impaired cognition, hyperactivity and social withdrawal (118). In particular, MAP6 deletion damage the cognitive function damaging the synaptic connectivity $(118,119)$. Studies on postmortem brains evidence that mRNA MAP6 levels are improved in prefrontal cortex of patients with schizophrenia. These studies together suggest an involvement of MAP6 with the onset of the schizophrenia (120), but further study will be necessary to point out the molecular mechanism.

\section{Therapeutic Drugs}

These studies indicate that microtubule regulators and PTM of MAPs are altered in psychiatric disorders. Some effects of these modifications can be rescued by the treatment with antidepressant drugs, neurosteroids like microtubule associated protein neurosteroidal pregnenolone (MAPREG), pregnenolone (PREG) and dehydroepiandrosterone (DHEA) were noticed to enhance the neuronal survival and improve long term memory (121). Binding of these neurosteroids that are 
synthetized in multiple glial cells $(122,123)$, promotes the neurite growth stimulating the MAP2 effect on MT polymerization (123-126). Neurosteroids improve the neuronal survival and have a neuroprotective effect, they can even stimulate neurogenesis in adult hippocampus improving the long term memory (127-129).

On the other hand, were noted that MT drugs have significant benefits on behaviour. In particular, lithium is a mood stabilizing drug used to treat bipolar disorder $(130,131)$. Studies revealed that the administration of lithium directly inhibit GSK-3, this reduces the phosphorylation of tau and MAP1B protein leading to MT remodelling (132-134). Modification of the cytoskeleton structure may help to stimulate the neuroplasticity in the brain areas interested by the mood disorder like hippocampus and amygdala (135).

\section{Conclusions}

As discussed herein, there is a convincing number of evidences that axonal transport troubles are involved in several neurodegenerative and neuropsychiatric disease. This issues in transport can be due in part or whole to the perturbed MT homeostasis, increase or decrease of microtubule stability. The focus of this review has been on how MT variations can alter axonal transport and there is a growing number of studies regarding MT defects in cell cultures or animal models of neurodegenerative or psychiatric disease. These observations have led to experiment MT stabilizing drugs, usually used in the treatment of cancer, on animal models of disease with good results. The challenge now is to develop and test new drugs that can support the treatment of neurodegenerative or psychiatric disease in human patients.

\section{Conflict of interest}

The authors declare that there is no conflict of interests regarding the publication of this paper.

\section{References}

1. Coles $\mathrm{CH}$, Bradke F. Coordinating neuronal actinmicrotubule dynamics. Curr Biol. 2015;25(15):R677-91.

2. Sainath R, Gallo G. Cytoskeletal and signaling mechanisms of neurite formation. Cell Tissue Res. 2015;359(1):267-78.

3. Mortal S, Iseppon F, Perissinotto A, D'Este E, Cojoc D, Napolitano LMR, et al. Actin waves do not boost neurite outgrowth in the early stages of neuron maturation. Front Cell Neurosci. 2017;11:402.

4. Howard J. Molecular motors: structural adaptations to cellular functions. Nature. 1997;389(6651):561-7.

5. Akhmanova A, Steinmetz MO. Tracking the ends: a dynamic protein network controls the fate of microtubule tips. Nat Rev Mol Cell Biol. 2008;9(4):309-22.

6. Barnes AP, Polleux F. Establishment of axon-dendrite polarity in developing neurons. Annu Rev Neurosci. 2009;32:347-81.

7. Kuijpers M, Hoogenraad CC. Centrosomes, microtubules and neuronal development. Mol Cell Neurosci. 2011;48(4):349-58.

8. Lipka J, Kuijpers M, Jaworski J, Hoogenraad CC. Mutations in cytoplasmic dynein and its regulators cause malformations of cortical development and neurodegenerative diseases. Biochem Soc Trans. 2013;41(6):1605-12.

9. Reiner O, Sapir T. LIS1 functions in normal development and disease. Curr Opin Neurobiol. 2013;23(6):951-6.

10. Howard J, Hyman AA. Growth, fluctuation and switching at microtubule plus ends. Nat Rev Mol Cell Biol. agosto 2009;10(8):569-74.

11. Alushin GM, Lander GC, Kellogg EH, Zhang R, Baker D, Nogales $E$. High-resolution microtubule structures reveal the structural transitions in $\alpha \beta$-tubulin upon GTP hydrolysis. Cell. 2014;157(5):1117-29.

12. Brouhard GJ, Rice LM. Microtubule dynamics: an interplay of biochemistry and mechanics. Nat Rev Mol Cell Biol. 2018;19(7):451-63.

13. Akhmanova A, Steinmetz MO. Control of microtubule organization and dynamics: two ends in the limelight. Nat Rev Mol Cell Biol. 2015;16(12):711-26.

14. Akhmanova A, Hoogenraad CC. Microtubule minus-endtargeting proteins. Curr Biol. 2015;25(4):R162-71.

15. Akhmanova A, Hoogenraad CC. Microtubule plus-endtracking proteins: mechanisms and functions. Curr Opin Cell Biol. 2005;17(1):47-54.

16. Dehmelt L, Halpain S. The MAP2/Tau family of microtubuleassociated proteins. Genome Biol. 2004;6(1):204.

17. Subramanian R, Kapoor TM. Building complexity: insights into self-organized assembly of microtubule-based architectures. Dev Cell. 2012;23(5):874-85.

18. Kollman JM, Merdes A, Mourey L, Agard DA. Microtubule nucleation by $\mathrm{y}$-tubulin complexes. Nat Rev Mol Cell Biol. 2011;12(11):709-21.

19. Sharp DJ, Ross JL. Microtubule-severing enzymes at the cutting edge. J Cell Sci. 2012;125(11):2561-9.

20. Hirokawa N, Niwa S, Tanaka Y. Molecular motors in neurons: transport mechanisms and roles in brain function, development, and disease. Neuron. 2010;68(4):610-38.

21. Hammond JW, Huang C-F, Kaech S, Jacobson C, Banker G, Verhey KJ. Posttranslational modifications of tubulin and the polarized transport of kinesin-1 in neurons. Mol Biol Cell. 2010;21(4):572-83.

22. Cooper JA. Cell biology in neuroscience: mechanisms of cell migration in the nervous system. J Cell Biol. 2013;202(5):725-34.

23. Cáceres A, Ye B, Dotti CG. Neuronal polarity: demarcation, growth and commitment. Curr Opin Cell Biol. 2012;24(4):547-53.

24. Flynn KC, Hellal F, Neukirchen D, Jacob S, Tahirovic S, Dupraz S, et al. ADF/Cofilin-mediated actin retrograde flow directs neurite formation in the developing brain. Neuron. 2012;76(6):1091-107.

25. Nakata T, Hirokawa N. Microtubules provide directional cues for polarized axonal transport through interaction with kinesin motor head. J Cell Biol. 2003;162(6):1045-55.

26. Nakata T, Niwa S, Okada Y, Perez F, Hirokawa N. Preferential binding of a kinesin-1 motor to GTP-tubulin-rich microtubules underlies polarized vesicle transport. The $\mathrm{J}$ Cell Biol. 2011;194(2):245-55.

27. Hellal F, Hurtado A, Ruschel J, Flynn KC, Laskowski CJ, Umlauf $\mathrm{M}$, et al. Microtubule stabilization reduces scarring and causes axon regeneration after spinal cord injury. Science. 2011;331(6019):928-31.

28. Ruschel J, Hellal F, Flynn KC, Dupraz S, Elliott DA, Tedeschi A, et al. Systemic administration of epothilone B promotes axon regeneration after spinal cord injury. Science. 2015;348(6232):347-52.

29. Hoogenraad CC, Bradke F. Control of neuronal polarity and plasticity - a renaissance for microtubules? Trends Cell Biol. 2009;19(12):669-76.

30. Bettencourt-Dias M, Glover DM. Centrosome biogenesis and function: centrosomics brings new understanding. Nat Rev Mol Cell Biol. 2007;8(6):451-63.

31. Kapitein LC, Hoogenraad CC. Building the neuronal 
microtubule cytoskeleton. Neuron. 2015;87(3):492-506.

32. Baas PW, Lin S. Hooks and comets: The story of microtubule polarity orientation in the neuron. Dev Neurobiol. 2011;71(6):403-18.

33. Kapitein LC, Hoogenraad CC. Which way to go? Cytoskeletal organization and polarized transport in neurons. Mol Cell Neurosci. 2011;46(1):9-20.

34. Kapitein LC, Schlager MA, Kuijpers M, Wulf PS, van Spronsen M, MacKintosh FC, et al. Mixed microtubules steer dynein-driven cargo transport into dendrites. Curr Biol. 2010;20(4):290-9.

35. Lu W, Gelfand VI. Moonlighting motors: kinesin, dynein, and cell polarity. Trends Cell Biol. 2017;27(7):505-14

36. Hirokawa N, Noda Y, Tanaka Y, Niwa S. Kinesin superfamily motor proteins and intracellular transport. Nat Rev Mol Cell Biol. 2009;10(10):682-96.

37. Bhabha G, Johnson GT, Schroeder CM, Vale RD. How dynein moves along microtubules. Trends Biochem Sci. 2016;41(1):94-105.

38. McNally FJ, Roll-Mecak A. Microtubule-severing enzymes: From cellular functions to molecular mechanism. J Cell Biol 2018;217(12):4057-69.

39. Roostalu J, Surrey T. Microtubule nucleation: beyond the template. Nat Rev Mol Cell Biol. 2017;18(11):702-10.

40. Bodakuntla S, Jijumon AS, Villablanca C, Gonzalez-Billault C, Janke C. Microtubule-associated proteins: structuring the cytoskeleton. Trends Cell Biol. 2019;29(10):804-19.

41. Kanai $\mathrm{Y}$, Takemura R, Oshima $\mathrm{T}$, Mori $\mathrm{H}$, Ihara $\mathrm{Y}$, Yanagisawa $\mathrm{M}$, et al. Expression of multiple tau isoforms and microtubule bundle formation in fibroblasts transfected with a single tau cDNA. J Cell Biol. 1989;109(3):1173-84.

42. Barlow S, Gonzalez-Garay ML, West RR, Olmsted JB, Cabral F. Stable expression of heterologous microtubuleassociated proteins (MAPs) in Chinese hamster ovary cells: evidence for differing roles of MAPs in microtubule organization. J Cell Biol. 1994;126(4):1017-29.

43. Wang Q, Crevenna AH, Kunze I, Mizuno N. Structural basis for the extended CAP-Gly domains of p150glued binding to microtubules and the implication for tubulin dynamics. Proc Natl Acad Sci U S A. 2014;111(31):11347-52.

44. Kalcheva N, Albala J, O'Guin K, Rubino H, Garner C, ShafitZagardo B. Genomic structure of human microtubuleassociated protein 2 (MAP-2) and characterization of additional MAP-2 isoforms. Proc Natl Acad Sci U S A. 1995;92(24):10894-8.

45. Matsunaga W, Miyata S, Kiyohara T. Redistribution of MAP2 immunoreactivity in the neurohypophysial astrocytes of adult rats during dehydration. Brain Res. 1999;829(1-2):7-17.

46. Al-Bassam J, Ozer RS, Safer D, Halpain S, Milligan RA. MAP2 and tau bind longitudinally along the outer ridges of microtubule protofilaments. J Cell Biol. 2002;157(7):1187-96.

47. Teng J, Takei Y, Harada A, Nakata T, Chen J, Hirokawa $\mathrm{N}$. Synergistic effects of MAP2 and MAP1B knockout in neuronal migration, dendritic outgrowth, and microtubule organization. J Cell Biol. 2001;155(1):65-76.

48. Roy S, Zhang B, Lee VM-Y, Trojanowski JQ. Axona transport defects: a common theme in neurodegenerative diseases. Acta Neuropathol. 2005;109(1):5-13.

49. Zhao $Y, M u X, D u$ G. Microtubule-stabilizing agents: New drug discovery and cancer therapy. Pharmacol Ther. 2016;162:134-43

50. Tsai RM, Miller Z, Koestler M, Rojas JC, Ljubenkov PA, Rosen $\mathrm{HJ}$, et al. Reactions to multiple ascending doses of the microtubule stabilizer TPI-287 in patients with Alzheimer Disease, progressive supranuclear palsy, and corticobasal syndrome: A randomized clinical trial. JAMA Neurol. 2020;77(2):215-24

51. Young JS, Hobbs JG, Bailes JE. The impact of traumatic brain injury on the aging brain. Curr Psychiatry Rep. 2016;18(9):81.

52. Saulle M, Greenwald BD. Chronic traumatic encephalopathy:
A review. Rehabil Res Pract. 2012;2012:816069.

53. Johnson VE, Stewart W, Smith DH. Axonal pathology in traumatic brain injury. Exp Neurol. 2013;246:35-43.

54. Tang-Schomer MD, Johnson VE, Baas PW, Stewart W, Smith DH. Partial interruption of axonal transport due to microtubule breakage accounts for the formation of periodic varicosities after traumatic axonal injury. Exp Neurol. 2012;233(1):364-72.

55. Tang-Schomer MD, Patel AR, Baas PW, Smith DH. Mechanical breaking of microtubules in axons during dynamic stretch injury underlies delayed elasticity, microtubule disassembly, and axon degeneration. FASEB J. 2010;24(5):1401-10.

56. McKee AC, Alosco ML, Huber BR. Repetitive head impacts and chronic traumatic encephalopathy. Neurosurg Clin N Am. 2016;27(4):529-35.

57. Gardner A, Iverson GL, McCrory P. Chronic traumatic encephalopathy in sport: a systematic review. Br J Sports Med. 2014;48(2):84-90.

58. Brizuela M, Blizzard CA, Chuckowree JA, Dawkins E, Gasperini RJ, Young KM, et al. The microtubule-stabilizing drug Epothilone $D$ increases axonal sprouting following transection injury in vitro. Mol Cell Neurosci. 2015;66:129-40.

59. Cross DJ, Garwin GG, Cline MM, Richards TL, Yarnykh $\mathrm{V}$, Mourad PD, et al. Paclitaxel improves outcome from traumatic brain injury. Brain Res. 2015;1618:299-308.

60. Chuckowree JA, Zhu Z, Brizuela M, Lee KM, Blizzard CA, Dickson TC. The microtubule-modulating drug Epothilone $D$ alters dendritic spine morphology in a mouse model of mild traumatic brain injury. Front Cell Neurosci. 2018;12:223

61. Ren Y, Jiang H, Hu Z, Fan K, Wang J, Janoschka S, et al. Parkin mutations reduce the complexity of neuronal processes in iPSC-derived human neurons. Stem Cells. 2015;33(1):68-78

62. Jiang $Q$, Ren $Y$, Feng J. Direct binding with histone deacetylase 6 mediates the reversible recruitment of parkin to the centrosome. J Neurosci. 2008;28(48):12993-3002.

63. Masrori P, Damme PV. Amyotrophic lateral sclerosis: a clinical review. Eur J Neurol. 2020;27(10):1918-29.

64. Bercier V, Hubbard JM, Fidelin K, Duroure K, Auer TO Revenu C, et al. Dynactin1 depletion leads to neuromuscular synapse instability and functional abnormalities. Mol Neurodegener. 2019;14(1):27.

65. Puls I, Jonnakuty C, LaMonte BH, Holzbaur ELF, Tokito M, Mann E, et al. Mutant dynactin in motor neuron disease. Nat Genet. 2003;33(4):455-6.

66. Moller A, Bauer CS, Cohen RN, Webster CP, De Vos KJ. Amyotrophic lateral sclerosis-associated mutant SOD1 inhibits anterograde axonal transport of mitochondria by reducing Miro1 levels. Hum Mol Genet. 2017;26(23):4668-79.

67. Zhang B, Tu P, Abtahian F, Trojanowski JQ, Lee VM-Y. Neurofilaments and orthograde transport are reduced in ventral root axons of transgenic mice that express human SOD1 with a G93A mutation. J Cell Biol. 1997;139(5):1307-15.

68. Williamson TL, Cleveland DW. Slowing of axonal transport is a very early event in the toxicity of ALS-linked SOD1 mutants to motor neurons. Nat Neurosci. 1999;2(1):50-6.

69. Fanara P, Banerjee J, Hueck RV, Harper MR, Awada M, Turner $\mathrm{H}$, et al. Stabilization of hyperdynamic microtubules is neuroprotective in amyotrophic lateral sclerosis. J Biol Chem. 2007;282(32):23465-72.

70. Kleele T, Marinković P, Williams PR, Stern S, Weigand EE, Engerer $\mathrm{P}$, et al. An assay to image neuronal microtubule dynamics in mice. Nat Commun. 2014;5(1):4827.

71. Huai J, Zhang Z. Structural properties and interaction partners of familial ALS-associated SOD1 mutants. Front Neurol. 2019;10:527.

72. Cohen TJ, Lee VMY, Trojanowski JQ. TDP-43 functions and pathogenic mechanisms implicated in TDP-43 proteinopathies. Trends Mol Med. 2011;17(11):659-67.

73. Magrané J, Cortez C, Gan W-B, Manfredi G. Abnormal 
mitochondrial transport and morphology are common pathological denominators in SOD1 and TDP43 ALS mouse models. Hum Mol Genet. 2014;23(6):1413-24.

74. Brunden KR, Yao Y, Potuzak JS, Ferrer NI, Ballatore C, James MJ, et al. The characterization of microtubulestabilizing drugs as possible therapeutic agents for Alzheimer's disease and related tauopathies. Pharmacol Res. 2011;63(4):341-51.

75. Brunden KR, Zhang B, Carroll J, Yao Y, Potuzak JS, Hogan $A-M L$, et al. Epothilone $D$ improves microtubule density, axonal integrity, and cognition in a transgenic mouse model of tauopathy. J Neurosci. 2010;30(41):13861-6.

76. Zhang B, Carroll J, Trojanowski JQ, Yao Y, Iba M, Potuzak JS, et al. The microtubule-stabilizing agent, Epothilone D, reduces axonal dysfunction, neurotoxicity, cognitive deficits, and Alzheimer-like pathology in an interventional study with aged tau transgenic mice. J Neurosci. 2012;32(11):3601-11.

77. Barten DM, Fanara P, Andorfer C, Hoque N, Wong PYA, Husted $\mathrm{KH}$, et al. Hyperdynamic microtubules, cognitive deficits, and pathology are improved in tau transgenic mice with low doses of the microtubule-stabilizing agent BMS241027. J Neurosci. 2012;32(21):7137-45.

78. Killinger BA, Moszczynska A. Epothilone D prevents binge methamphetamine-mediated loss of striatal dopaminergic markers. J Neurochem. 2016;136(3):510-25.

79. Brunden KR, Gardner NM, James MJ, Yao Y, Trojanowski $J Q$, Lee VM-Y, et al. MT-stabilizer, dictyostatin, exhibits prolonged brain retention and activity: potential therapeutic implications. ACS Med Chem Lett. 2013;4(9):886-9.

80. Makani V, Zhang B, Han H, Yao Y, Lassalas P, Lou K, et al. Evaluation of the brain-penetrant microtubule-stabilizing agent, dictyostatin, in the PS19 tau transgenic mouse model of tauopathy. Acta Neuropathol Commun. 2016;4(1):106.

81. Ballatore $C$, Brunden KR, Huryn DM, Trojanowski JQ, Lee VM-Y, Smith AB. Microtubule stabilizing agents as potential treatment for Alzheimer's Disease and related neurodegenerative tauopathies. J Med Chem. 2012;55(21):8979-96.

82. Brunden KR, Trojanowski JQ, Smith AB, Lee VM-Y, Ballatore $C$. Microtubule-stabilizing agents as potentia therapeutics for neurodegenerative disease. Bioorg Med Chem. 2014;22(18):5040-9.

83. Bianchi M, Shah AJ, Fone KCF, Atkins AR, Dawson LA, Heidbreder CA, et al. Fluoxetine administration modulates the cytoskeletal microtubular system in the rat hippocampus. Synapse. 2009;63(4):359-64.

84. Piubelli C, Carboni L, Becchi S, Mathé AA, Domenici E. Regulation of cytoskeleton machinery, neurogenesis and energy metabolism pathways in a rat gene-environment model of depression revealed by proteomic analysis. Neuroscience. 2011;176:349-80.

85. Prabakaran S, Swatton JE, Ryan MM, Huffaker SJ, Huang JT-J, Griffin JL, et al. Mitochondrial dysfunction in schizophrenia: evidence for compromised brain metabolism and oxidative stress. Mol Psychiatry. 2004;9(7):684-97, 643

86. Kang HJ, Voleti B, Hajszan T, Rajkowska G, Stockmeier CA Licznerski $P$, et al. Decreased expression of synapse-related genes and loss of synapses in major depressive disorder. Nat Med. 2012;18(9):1413-7.

87. Verhey KJ, Gaertig J. The tubulin code. Cell Cycle 2007;6(17):2152-60.

88. Magiera MM, Janke C. Post-translational modifications of tubulin. Curr Biol. 2014;24(9):R351-4

89. Hubbert C, Guardiola A, Shao R, Kawaguchi Y, Ito A, Nixon $A$, et al. HDAC6 is a microtubule-associated deacetylase. Nature. 2002;417(6887):455-8.

90. Fuchikami M, Thomas A, Liu R, Wohleb ES, Land BB DiLeone RJ, et al. Optogenetic stimulation of infralimbic PFC reproduces ketamine's rapid and sustained antidepressant actions. Proc Natl Acad Sci U S A. 2015;112(26):8106-11.

91. Fukada M, Hanai A, Nakayama A, Suzuki T, Miyata N,
Rodriguiz RM, et al. Loss of deacetylation activity of Hdac6 affects emotional behavior in mice. PLOS ONE. 2012;7(2):e30924.

92. Liu R, Dang W, Du Y, Zhou Q, Jiao K, Liu Z. SIRT2 is involved in the modulation of depressive behaviors. Sci Rep. 2015;5(1):8415.

93. Yang C, Wang G, Wang H, Liu Z, Wang X. Cytoskeletal alterations in rat hippocampus following chronic unpredictable mild stress and re-exposure to acute and chronic unpredictable mild stress. Behav Brain Res. 2009;205(2):518-24.

94. Lang B, Pu J, Hunter I, Liu M, Martin-Granados C, Reilly TJ, et al. Recurrent deletions of ULK4 in schizophrenia: a gene crucial for neuritogenesis and neuronal motility. J Cell Sci. 2014;127(3):630-40.

95. Coffey ET. Nuclear and cytosolic JNK signalling in neurons. Nat Rev Neurosci. 2014;15(5):285-99

96. Alexander JE, Hunt DF, Lee MK, Shabanowitz J, Michel $\mathrm{H}$, Berlin SC, et al. Characterization of posttranslational modifications in neuron-specific class III beta-tubulin by mass spectrometry. Proc Natl Acad Sci U S A. 1991;88(11):4685-9.

97. Matten WT, Aubry M, West J, Maness PF. Tubulin is phosphorylated at tyrosine by pp60c-src in nerve growth cone membranes. J Cell Biol. 1990;111(5):1959-70.

98. Greenberger L.M., Loganzo F. (2008) Destabilizing Agents. In: Fojo T. (eds) The Role of Microtubules in Cell Biology, Neurobiology, and Oncology. Cancer Drug Discovery and Development. Humana Press. https://doi.org/10.1007/978-159745-336-3 10

99. Corena-McLeod M, Walss-Bass C, Oliveros A, Villegas AG, Ceballos C, Charlesworth CM, et al. New model of action for mood stabilizers: phosphoproteome from rat prefrontal cortex synaptoneurosomal preparations. PLOS ONE. 2013;8(5):e52147.

100. Janke C. The tubulin code: Molecular components, readout mechanisms, and functions. J Cell Biol. 2014;206(4):461-72.

101. Ikegami K, Heier RL, Taruishi M, Takagi H, Mukai M, Shimma $S$, et al. Loss of $\alpha$-tubulin polyglutamylation in ROSA22 mice is associated with abnormal targeting of KIF1A and modulated synaptic function. Proc Natl Acad Sci U S A. 2007;104(9):3213-8.

102. Rajkumar AP, Christensen JH, Mattheisen M, Jacobsen I, Bache I, Pallesen J, et al. Analysis of $t(9 ; 17)(q 33.2 ; q 25.3)$ chromosomal breakpoint regions and genetic association reveals novel candidate genes for bipolar disorder. Bipolar Disord. 2015;17(2):205-11.

103. Fullston $T$, Gabb B, Callen D, Ullmann R, Woollatt E, Bain S et al. Inherited balanced translocation $\mathrm{t}(9 ; 17)(\mathrm{q} 33.2 ; \mathrm{q} 25.3)$ concomitant with a 16p13.1 duplication in a patient with schizophrenia. Am J Med Genet B Neuropsychiatr Genet. 2011;156(2):204-14.

104. Fukushima N, Furuta D, Hidaka Y, Moriyama R, Tsujiuchi T. Post-translational modifications of tubulin in the nervous system. J Neurochem. 2009;109(3):683-93.

105. Bosch Grau M, Gonzalez Curto G, Rocha C, Magiera MM Marques Sousa P, Giordano T, et al. Tubulin glycylases and glutamylases have distinct functions in stabilization and motility of ependymal cilia. J Cell Biol. 2013;202(3):441-51.

106. Marley A, Zastrow M von. A simple cell-based assay reveals that diverse neuropsychiatric risk genes converge on primary cilia. PLOS ONE. 2012;7(10):e46647.

107. Blanpied TA, Ehlers MD. Microanatomy of dendritic spines: emerging principles of synaptic pathology in psychiatric and neurological disease. Biol Psychiatry. 2004;55(12):1121-7.

108. Penzes $P$, Cahill ME, Jones KA, VanLeeuwen J-E, Woolfrey $\mathrm{KM}$. Dendritic spine pathology in neuropsychiatric disorders. Nat Neurosci. 2011;14(3):285-93.

109. Kaufmann WE, MacDonald SM, Altamura CR. Dendritic cytoskeletal protein expression in mental retardation: an immunohistochemical study of the neocortex in Rett 
syndrome. Cereb Cortex. 2000;10(10):992-1004.

110. Duman EA, Canli T. Influence of life stress, 5-HTTLPR genotype, and SLC6A4 methylation on gene expression and stress response in healthy Caucasian males. Biol Mood Anxiety Disord. 2015;5:2.

111. Varidaki A, Mohammad H, Coffey ET. Chapter 5 - Molecular Mechanisms of Depression. In: Frodl T, curatore. Systems Neuroscience in Depression [Internet]. San Diego: Academic Press; 2016 [citato 5 febbraio 2021]. pag. 14378. Disponibile su: https://www.sciencedirect.com/science/ article/pii/B9780128024560000054

112. Gold PW. The organization of the stress system and its dysregulation in depressive illness. Mol Psychiatry. 2015;20(1):32-47.

113. Shelton MA, Newman JT, Gu H, Sampson AR, Fish KN, MacDonald ML, et al. Loss of microtubule-associated protein 2 immunoreactivity linked to dendritic spine loss in schizophrenia. Biol Psychiatry. 2015;78(6):374-85.

114. Harada A, Teng J, Takei Y, Oguchi K, Hirokawa N. MAP2 is required for dendrite elongation, PKA anchoring in dendrites, and proper PKA signal transduction. J Cell Biol. 2002;158(3):541-9.

115. Law AJ, Weickert CS, Hyde TM, Kleinman JE, Harrison PJ. Reduced spinophilin but not microtubule-associated protein 2 expression in the hippocampal formation in schizophrenia and mood disorders: molecular evidence for a pathology of dendritic spines. Am J Psychiatry. 2004;161(10):1848-55.

116. Bosc C, Cronk JD, Pirollet F, Watterson DM, Haiech J, Job D, et al. Cloning, expression, and properties of the microtubule-stabilizing protein STOP. Proc Natl Acad Sci U S A. 1996;93(5):2125-30.

117. Lefèvre J, Savarin P, Gans P, Hamon L, Clément M-J, David $\mathrm{M}-\mathrm{O}$, et al. Structural basis for the association of MAP6 protein with microtubules and its regulation by calmodulin. $\mathrm{J}$ Biol Chem. 2013;288(34):24910-22.

118. Fournet V, Schweitzer A, Chevarin C, Deloulme J-C, Hamon M, Giros B, et al. The deletion of STOP/MAP6 protein in mice triggers highly altered mood and impaired cognitive performances. J Neurochem. 2012;121(1):99-114.

119. Gozes I. Microtubules, schizophrenia and cognitive behavior: preclinical development of davunetide (NAP) as a peptide-drug candidate. Peptides. 2011;32(2):428-31.

120. Shimizu H, Iwayama $Y$, Yamada K, Toyota T, Minabe $Y$, Nakamura K, et al. Genetic and expression analyses of the STOP (MAP6) gene in schizophrenia. Schizophr Res. 2006;84(2-3):244-52.

121. Plassart-Schiess E, Baulieu E-E. Neurosteroids: recent findings. Brain Res Brain Res Rev. 2001;37(1):133-40.

122. Tsutsui K, Ukena K, Usui M, Sakamoto H, Takase M. Novel brain function: biosynthesis and actions of neurosteroids in neurons. Neurosci Res. 2000;36(4):261-73.
123. Fontaine-Lenoir V, Chambraud B, Fellous A, David S, Duchossoy Y, Baulieu E-E, et al. Microtubule-associated protein 2 (MAP2) is a neurosteroid receptor. Proc Natl Acad Sci U S A. 2006;103(12):4711-6.

124. Cardounel A, Regelson W, Kalimi M. Dehydroepiandrosterone protects hippocampal neurons against neurotoxin-induced cell death: mechanism of action2. Proc Soc Exp Biol Med. 1999;222(2):145-9.

125. Marx CE, Jarskog LF, Lauder JM, Gilmore JH, Lieberman JA, Morrow AL. Neurosteroid modulation of embryonic neuronal survival in vitro following anoxia. Brain Res. 2000;871(1):104-12.

126. Murakami K, Fellous A, Baulieu E-E, Robel P. Pregnenolone binds to microtubule-associated protein 2 and stimulates microtubule assembly. Proc Natl Acad Sci U S A. 2000;97(7):3579-84.

127. Roberts E, Bologa L, Flood JF, Smith GE. Effects of dehydroepiandrosterone and its sulfate on brain tissue in culture and on memory in mice. Brain Res. 1987;406(1):357-62.

128. Flood JF, Morley JE, Roberts E. Memory-enhancing effects in male mice of pregnenolone and steroids metabolically derived from it. Proc Natl Acad Sci U S A. 1992;89(5):1567-71.

129. Karishma KK, Herbert J. Dehydroepiandrosterone (DHEA) stimulates neurogenesis in the hippocampus of the rat, promotes survival of newly formed neurons and prevents corticosterone-induced suppression. Eur J Neurosci. 2002;16(3):445-53.

130. Drago A, Crisafulli C, Sidoti A, Calabrò M, Serretti A. The microtubule-associated molecular pathways may be genetically disrupted in patients with Bipolar Disorder. Insights from the molecular cascades. J Affect Disord. 2016;190:429-38.

131. Beurel E, Song L, Jope RS. Inhibition of glycogen synthase kinase-3 is necessary for the rapid antidepressant effect of ketamine in mice. Mol Psychiatry. 2011;16(11):1068-70.

132. Goold RG, Owen R, Gordon-Weeks PR. Glycogen synthase kinase 3beta phosphorylation of microtubule-associated protein $1 \mathrm{~B}$ regulates the stability of microtubules in growth cones. J Cell Sci. 1999;112(19):3373-84.

133. Grimes CA, Jope RS. CREB DNA binding activity is inhibited by glycogen synthase kinase- $3 \beta$ and facilitated by lithium. $J$ Neurochem. 2001;78(6):1219-32.

134. Bélanger D, Farah CA, Nguyen MD, Lauzon M, Cornibert $S$, Leclerc $N$. The projection domain of MAP2b regulates microtubule protrusion and process formation in Sf9 cells. J Cell Sci. 2002;115(7):1523-39.

135. Cole AR. Glycogen synthase kinase 3 substrates in mood disorders and schizophrenia. The FEBS Journal. 2013;280(21):5213-27. 